\title{
TRASDISCIPLINARIDADE NO ENSINO E PESQUISA DA GERAÇÃO DE FORMAS ARQUITETÔNICAS
}

\section{TRASDISCIPLINARIEDAD EN LA ENSEÑANZA E INVESTIGACIÓN DE LA GENERACIÓN DE FORMAS ARQUITECTÓNICAS}

\section{CROSSDISCIPLINARITY IN THE TEACHING AND RESEARCH OF THE ARCHITECTURAL FORMS GENERATION}

\section{PERÍES, LUCAS}

Doutor, Arquiteto, Universidad Nacional de Córdoba. Facultad de Arquitectura, Urbanismo y Diseño. Cátedra de Morfología II B. E-mail: lucasperies@unc.edu.ar

\section{BARRAUD, SILVINA}

Mestre, Arquiteta, Universidad Nacional de Córdoba. Facultad de Arquitectura, Urbanismo y Diseño. Cátedra de Morfología II B. E-mail: silvina.barraud@unc.edu.ar

\section{RESUMO}

Na pesquisa apresentada neste artigo, é abordada a relação específica entre as ordens geométricas e a geração da forma arquitetônica contemporânea, com a intenção de fornecer ferramentas generativas que participem ativamente durante o processo de ensino e aprendizagem da morfologia. A disciplinaridade cruzada ou unidirecional é apresentada como uma condição das relações entre disciplinas e como uma abordagem que apela à procura de estruturas conceituais e técnicas em outros campos do conhecimento, aquelas com potencial para a sua transferência para a arquitetura. Neste contexto, a mistura é produzida em leis, princípios, ferramentas e técnicas externas para a produção de conhecimento disciplinar integral. A atenção é colocada na física, matemática aplicada, hidrografia, ceraunologia (parte da meteorologia que estuda os raios e seus fenômenos), neurologia e botânica, caracterizadas por favorecer a identificação de padrões com potencial de ensaio e sistematização através de práticas de indagação que contribuem para a geração de formas arquitetônicas. A natureza da pesquisa é qualitativa e o seu desenho metodológico é bibliográfico, exploratório e experimental. Numa primeira fase, é realizada a pesquisa documental e é especificada a classificação dos casos multidisciplinares. Numa segunda instância, o trabalho é colocado no âmbito da pesquisa projetual, e é experimentado a fim de produzir conhecimentos através da utilização do projeto e do seu processo de construção.

PALAVRAS-CHAVE: morfologia; processo de design; projeto; disciplinas.

\section{RESUMEN}

En la investigación que se expone en este artículo, se afronta la relación específica entre los órdenes geométricos y la generación de la forma arquitectónica contemporánea, con intención de aportar herramientas generativas que participen de modo activo durante el proceso de enseñanza y aprendizaje de la morfología. La disciplinaridad cruzada o unidireccional, se presenta como condición de relaciones entre disciplinas y como enfoque, que apela a la búsqueda de estructuras conceptuales y técnicas en otros campos del conocimiento, aquellas con potencial para su traspaso a la arquitectura. En ese contexto, la mixtura se produce en leyes, principios, herramientas y técnicas externas para producción de conocimiento integral disciplinar. La atención se coloca en física, matemática aplicada, hidrografía ceraunología, neurología y botánica, caracterizadas por favorecer la identificación de patrones con potencial para su ensayo y sistematización por medio de prácticas de indagación contributivas de la generación de formas arquitectónicas. El carácter de la investigación es cualitativo y su diseño metodológico es bibliográfico exploratorio y experimental. En una primera etapa, se despliega la búsqueda documental y se concreta la clasificación de casos multidisciplinares. En una segunda instancia, el trabajo se sitúa en el marco de la investigación proyectual, y se experimenta para producir conocimientos mediante el uso del proyecto y de su proceso de construcción

PALABRAS CLAVE: morfología; proceso de diseño; proyecto; disciplinas.

\section{ABSTRACT}

In the research presented in this article, the specific relationship between geometric orders and the generation of contemporary architectural form is addressed, with the intention of providing generative tools that actively participate during the morphology teaching and learning process. Cross or unidirectional disciplinarity is presented as a condition of relations between disciplines and as an approach, which appeals to the search for conceptual and technical structures in other fields of knowledge, those with potential for transfer to architecture. In this context, the mixture is produced in external laws, principles, tools and techniques for the production of comprehensive disciplinary knowledge. The focus is placed on: physics, applied mathematics, hydrography, ceraunology, neurology and botany, characterized by favoring the identification of patterns with potential for their testing and systematization through contributory inquiry practices of the generation of architectural forms. The character of the research is qualitative and its methodological design is bibliographic, exploratory and experimental. In a first stage, the documentary search is deployed and the classification of multidisciplinary cases is specified. In a second instance, the work is located within the framework of project research, and is experimented to produce knowledge through the use of the project and its construction process. 


\section{INTRODUÇÃO}

A pesquisa, da qual este artigo é derivado, explora princípios de ordem alternativos aos comumente empregados no ensino da morfologia arquitetônica. Como expressa Litwin (2016, p. 203), o objetivo é “...avançar em uma construção teórica que permita a análise das propostas pedagógicas usadas a partir de outro ponto de vista"; e, nesse marco, terminam sendo de interesse as estruturas conceituais e técnicas que estão alojadas em campos externos do conhecimento, com o potencial para sua transferência para a própria disciplina. O foco está na física, matemática aplicada, hidrografia, ceraunologia, neurologia e botânica. $O$ sentido de explorar nestas disciplinas é orientado para identificar padrões com potencial de ensaio e sistematização através de práticas que favoreçam a pesquisa e contribuam para a geração de formas arquitetônicas. A busca de antecedentes transcende os conhecimentos recentes, e é considerado oportuno rever casos do passado, particularmente aqueles que podem ser atualizados para fornecer novas chaves inéditas para o contexto disciplinar particular da morfologia arquitetônica. Com respeito a este último, são de grande interesse, e em termos ilustrativos globais, os volumes editados por Martineau (2014/2016): Quadrivium, Designa, Sciencia, Trivium, Geomancia e Megalitos.

Em termos de metodologia, o desafio proposto é abordado através do enfoque da disciplinaridade cruzada, que no tecido das relações disciplinares é um dos mais recentes. Em 1970, Piaget abordou o termo "transdisciplinar" e, desde então, sete escalas foram definidas como alternativas e possibilidades de relações disciplinares: transdisciplinar, interdisciplinar, polidisciplinar, pluridisciplinar, multidisciplinar, interdisciplinar e disciplinar. Desde o final dos anos 1980, surgiu o conceito de "disciplinaridade cruzada", que foi recentemente definido por Margery Bertoglia (2019) como um ato que implica que uma disciplina seja "vista" a partir da perspectiva de outra.

O trabalho de pesquisa está diretamente ligado ao ensino; os ensaios e comprovações são realizados através da prática de exercícios projetuais acadêmicos no âmbito da cadeira de Morfologia II B, no curso de graduação em Arquitetura da Facultad de Arquitectura, Urbanismo y Diseño da Universidad Nacional de Córdoba (FAUDDUNC).

\section{A TRASDISCIPLINA}

A trasdisciplinariedad (em espanhol), também chamada disciplinaridade cruzada ou unidirecional, como a qualificam Di Castri e Hadley (1986), há mais de trinta anos deriva do termo na língua inglesa: crossdisciplinarity. Não há uma tradução direta para o espanhol, por isso se decide aplicar o prefixo espanhol tras-, com seu significado "através", e em distinção da transdisciplinaridade, que usa o prefixo trans-, com significado "do outro lado de". A partir dessa primeira desagregação esclarecedora, os termos podem ser diferenciados da seguinte forma:

TRANS-disciplina significa que a solução de um problema, nos campos científico e projetuais, resulta da origem e estabelecimento de axiomas e métodos que são utilizados conjuntamente por diferentes disciplinas, com relações de construção conjunta, com um ponto de vista epistemológico, com razões e objetivos únicos, aqueles que orientam os esforços de todos os seus participantes (provenientes ou não de âmbitos acadêmicas) sobre um tema comum. Esta é, por exemplo, a ação conjunta dos profissionais de arquitetura, biologia e geologia para realizar um estudo paisagístico. Uma equipe transdisciplinar é holística e procura relacionar as disciplinas em um todo coerente ou unificado. Nicolescu (1996, p. 11) define este processo como "uma alegre transgressão das fronteiras entre disciplinas, especialmente no campo do ensino, para superar o pluralismo e a interdisciplinaridade". Isso permite superar a fragmentação do conhecimento, além do enriquecimento das disciplinas com diferentes conhecimentos (multidisciplina) e a troca de conhecimentos epistemológicos e de métodos científicos dos saberes (interdisciplina). Seu valor reside na interpretação de uma disciplina a partir da perspectiva de outra; isto se refere ao fato de que envolve uma combinação de conhecimentos e métodos, com base em uma síntese de suas abordagens. Esta teoria é um produto da reflexão filosófica renovada pelas descobertas da física quântica quase desde o início e ao longo do século $\mathrm{XX}$, assim como, mais recentemente, pelo advento das novas ciências da informação e pelo desenvolvimento da teoria geral dos sistemas, a partir da segunda metade do século passado. Portanto, é um "processo de reflexão contínuo e inacabado" (SARQUIS \& BUGANZA, 2009, p. 44). O desafio da transdisciplina "(...) é quebrar a realidade fragmentada pela disciplinarização do conhecimento, para criar um conjunto de conhecimento que transcenda qualquer disciplina" (SÁNCHEZ YUSTOS, 2014, p. 13).

TRAS-disciplina significa que a solução de um problema, nos campos científicos e de projeto, resulta do cruzamento ou do uso de axiomas e métodos de uma disciplina por outra, com diferentes razões e objetivos sobre um assunto não necessariamente comum, como um modo de interpretação ou reinterpretação por ocasião de um objetivo disciplinar específico. É usado para substanciar os aspectos de um problema em uma 
disciplina, a partir dos termos de outra. É, por exemplo, a ação dos profissionais da arquitetura para realizar um estudo disciplinar a partir dos axiomas da geologia. Uma equipe trasdisciplinar é reducionista - como um antônimo de holístico-, e pode ser monodisciplinar, procura produzir conhecimento através de saberes que foram desenvolvidos previamente em outras disciplinas. A construção trasdisciplinar parte da análise de um objeto de conhecimento geral e estabelece a partir disto a possível concorrência disciplinar para a produção do próprio conhecimento. Refere-se a um processo de trânsito que articula, integra e relaciona conhecimentos, transcendendo análises especializadas ou particulares para o mesmo campo de conhecimento. Neste marco conceitual, as disciplinas atuam em termos de cruzamento e como meio de "através de" determinado conhecimento externo para desenvolver a geração do conhecimento interno. Com esta abordagem cultural e científica, observamos as ações e interações entre os diferentes conhecimentos, na transferência de conceitos e experiências de uma disciplina para outra.

A disciplinaridade cruzada é caracterizada por De Pablos Pons (2006, p. 84) como “...uma ponte de trânsito entre diferentes compartimentos do saber, o que permite um conhecimento mais abrangente por ser interativo"; por outro lado, o conceito implica uma abordagem baseada em uma "posição de forças" (1994, p. 74); a partir disso, a possibilidade de comunicação não é equilibrada, já que certas forças são apresentadas de forma dominante sobre as outras e as tracionam. A disciplina que gera a travessia assume a tração das outras e define esse "trânsito", em determinadas circunstâncias, a partir de níveis prévios à interdisciplinaridade. Também na linha da "disciplinaridade cruzada", Arnaud, Maniez e Renner (2012) referem-se à ideia de blend, termo inglês que pode ser traduzido como mistura e nesse sentido a mistura ocorre em categorias conceituais, leis, instrumentos, ferramentas e técnicas para a produção de conhecimento integral.

\section{A TRASDISCIPLINA NA ARQUITETURA}

Em qualquer arte [e na arquitetura], os meios de regeneração devem ser externos. Eles vêm quase sempre de fora do âmbito da alta cultura e são remotos no espaço e no tempo. Os impulsos de renovação, se não um apelo direto à natureza, vêm da arte arcaica. (MUÑOZ, 2010, p. 11).

Com este parágrafo, que é usado como uma citação introdutória para esta seção, começa o livro La mirada del otro (MUÑOZ, 2010); e é coletado com a intenção de expressar o propósito e o sentido preciso que é perseguido com a abordagem da transdisciplina. Quintanilla e Vidal afirmam que "um determinado pesquisador se considerará um especialista em um determinado campo científico, mas para trabalhar nessa área ele precisa dominar e ser capaz de usar métodos e resultados de várias disciplinas diferentes" (2000, p. 54). ${ }^{1}$

A complexidade atual exige a abordagem e a valorização dos fenômenos de forma interligada com amplas perspectivas, e a morfologia arquitetônica não ignora a necessidade de incorporar essa visão, enquanto que, como disciplina, ela também forma parte de outras. Com referência específica ao campo da morfologia, Doberti (2008) descreve a relação entre forma e interdisciplina e afirma que: "A Morfologia Primeira trata da lógica da Conformação, concentra-se nela e trata apenas dos passos necessários para alcançar a Forma". (p. 108). A essa morfologia, especificamente, a caracteriza como "filosófica" (p. 108). Doberti também explica que "a forma é um gerador de disciplinas e um facilitador da interdisciplina, assim como um facilitador da penetração de limites" (p. 109).

$\mathrm{Na}$ história da arquitetura, principalmente desde o século passado, são reconhecidos múltiplos antecedentes do campo educacional que exploram a transferência de saberes no desenvolvimento de processos de design; a Bauhaus (1919-1933), a Escola de Artes e Ofícios da Basiléia (1944-1964) ou o Instituto de Design da Universidade Católica de Córdoba (1990-2015) são três exemplos representativos e vanguardistas que produziram contribuições paradigmáticas. No ambiente profissional, também são desenvolvidos processos de projeto que são influenciados pelo conhecimento multidisciplinar, tanto de forma racional e consciente como por intuição criativa. Há profissionais que envolvem relações com outros campos do conhecimento, desde o estabelecimento de figuras metafóricas, por analogias ou pela transferência de métodos ou técnicas precisas. A arquiteta Banchio (2012), no seu trabalho final de pós-graduação, realiza o estudo de antecedentes relevantes da cultura contemporânea: Peter Eisenman e o vínculo com as artes plásticas no projeto da Casa VI Cornwall - a arte conceitual - Steven Holl com a música na Casa Stretto - a música para cordas, percussão e celesta - Toyo Ito com a biologia na Mediateca de Sendai - a algologia - e Herzog \& de Meuron com a fotografia para o Museu de Young - a imagem digital - . "O trabalho de pesquisa realiza uma jornada analítica através do processo projetual dos quatro casos, avaliando estratégias inovadoras, teorias científicas e conexões multidisciplinares" (BANCHIO, 2012, p. 46). São obras nas quais 
são reconhecidas práticas concretas e profundas, desde a concepção do projeto até a obra construída e nas quais são produzidas configurações de morfologia singular e original.

A pesquisa apresentada neste artigo trata da relação específica entre as ordens geométricas e a origem da forma arquitetônica contemporânea com a intenção de fornecer ferramentas generativas que participem ativamente durante o processo de ensino e aprendizagem da morfologia, com base na busca em outros campos disciplinares de leis e princípios que favoreçam o desenvolvimento de traçados geométricos ordenadores. A disciplinaridade cruzada permite uma abordagem concreta dessas relações e, apesar de ser baseada em uma relação de forças na qual uma das disciplinas tem domínio sobre as outras, favorece a captura da riqueza e promove a interação entre os subsistemas de conhecimento das disciplinas envolvidas, para seu próprio benefício.

\section{METODOLOGIA}

A pesquisa é de natureza qualitativa e o esquema metodológico é bibliográfico, exploratório e experimental. Em primeira instância, há uma fase documental a partir da busca e classificação de casos multidisciplinares. Em uma segunda instância, o trabalho está situado no âmbito da pesquisa projetual, no qual é indagada para gerar conhecimentos através do uso do próprio projeto e de seu processo de construção.

A tarefa aprofunda o estudo de projetos em experiências didáticas com estudantes do segundo nível do curso de Arquitetura. Como afirma Litwin (2016, p. 203): "A pesquisa na sala de aula pressupõe um processo de construção teórica que permite aos professores refletir mais profundamente sobre as atividades que estão sendo promovidas, suas consequências e suas implicações". Neste caso, as implicações dizem respeito ao processo de projeto arquitetônico. Estes trabalhos são de transferência e retroalimentação direta à pesquisa e são complementados com os conhecimentos trasdisciplinares. $\mathrm{O}$ trabalho é baseado em uma abordagem do método fenomenológico, enfatizando a observação de eventos e fenômenos ocorridos no ato de experimentar e projetar.

As práticas experimentais são realizadas no âmbito de um curso de morfologia, sob a direção dos assinantes deste artigo. O objetivo da proposta pedagógica e do projeto científico é combinar ensino e pesquisa em um sistema simultâneo de produção e retroalimentação. O conhecimento é gerado em ambas as áreas, em diferentes instâncias do trabalho, o que permite concentrar esforços e enriquecer os resultados. E como Pokropek expressa "(...) aprende-se a fazer projetos projetando" (2020, p .115), e da mesma forma, aprendese a pesquisar pesquisando. Com relação ao vínculo entre o exercício projetual e a pesquisa-criação PavaGómez, Betancur-Villegas e Páez-Calvo afirmam que “(...) estas noções devem ser articuladas de forma tangível para dar conta da tensão que torna possível sua determinação". (2018, p. 90). Por outro lado, esta configuração didática se opõe ao modelo clássico de transmissão de dogmas e repetição de exercícios com soluções pré-estabelecidas, e é por isso que a experimentação de projetos é enfrentada.

\section{EXPERIMENTAÇÃO PROJETUAL}

A fase de pesquisa documental tem sua transferência e implementação a partir da experimentação acadêmica. "Experimentar em circunstâncias projetuais consiste em confirmar ou refutar hipóteses, como no método científico clássico; neste caso, através do exercício, o teste, o ensaio e o estudo do projeto e seu processo" (PERIES, 2017, p. 535). Neste sentido, é interessante realizar ensaios que transfiram os princípios detectados na física, matemática aplicada, hidrografia, ceraunología, neurologia, botânica, com potencial para sua aplicação no processo de projeto arquitetônico. A produção de formas toma como iniciador uma série de padrões biológicos, hidrológicos, meteorológicos, matemáticos, mecânicos e acústicos.

Como modalidade operativa, para abordar as teorias e padrões indicados, em referência às diferentes linhas temáticas que são enfrentadas a partir do cruzamento disciplinar, cada uma delas está a cargo de um professor-pesquisador, que trabalha de forma concentrada - a partir da realização de estudos e práticas específicas -, juntamente com uma equipe de estudantes. Como este é um contexto universitário massivo, em cada ciclo anual, trabalhamos com oito comissões compostas em média por 55 estudantes.

O corpo estudantil participa da co-pesquisa, do diálogo e do trabalho conjunto (POKROPEK, 2020), com uma metodologia que é abordada a partir da observação direta e do registro dos fenômenos e processos que ocorrem. Tendo em vista a apresentação dos diferentes temas - como base conceitual - e as instruções de trabalho - como base operacional - a abordagem coletiva das hipóteses de trabalho é desenvolvida, a fim de experimentar em relação a elas, a partir da busca de respostas e comprovações. Esta estratégia científico-didática, na qual os saberes são "misturados", de acordo com o significado dado à conceituação de 
"disciplinaridade cruzada" por Arnaud, Maniez e Renner (2012), permite a construção do conhecimento a partir da própria experimentação nas aulas-laboratório. A seguir, apresenta-se um resumo das linhas temáticas estudadas e, a fim de simplificar seu tratamento, algumas delas são agrupadas por afinidade ou similaridade.

\section{Lei construtural e sistemas de ramificação}

Em primeiro lugar, os conhecimentos de física, geografia, medicina e biologia convergem de forma associada; para o estudo e aplicação da lei construtural e dos sistemas de ramificação - ambos vêm do estudo das formas ou dos fenômenos naturais - .

No caso da lei construtural, a mesma é abordada do ponto de vista hidrográfico, a fim de viabilizar a comparação com os sistemas de ramificação; e é particularmente questionada nos padrões de drenagem dos seguintes tipos: dendrítica, paralela, treliça, treliçada ou trellis, retangular, radial e anular. As leis propostas por Horton (1945) e Strahler (1952) são analisadas como um sistema de regulamentação baseado no estudo das bacias hidrográficas que permite a definição da complexidade de suas ramificações. A lei de construção (BEJAN, 1996) rege os sistemas dinâmicos, permite tornar visível a evolução natural de certos processos e é aplicada particularmente nos fluxos. Os sistemas de ramificação se manifestam na natureza através de estruturas de crescimento com lógica de ramificação. De particular interesse os que são abordados pela ceraunología (sistemas de raios elétricos), neurologia (sistemas neuronais) e botânica (sistemas vegetais).

A transferência destes princípios para a prática experimental é desenvolvida a partir da observação direta dos fenômenos ou por meio de imagens, como ferramentas substitutas. A construção de modelos de simulação é outro recurso utilizado, principalmente para os ensaios com sistemas de fluidos dinâmicos. A dedução e compreensão de cada sistema permite realizar decodificações abstratas a partir da representação de estruturas geométricas, que são exibidas por meio de linhas de diferentes espessuras e que se estendem em várias direções. Os padrões e princípios generativos desta linha temática, por sua natureza intrínseca, proporcionam precisão e clareza de ordem geométrica desde o início da experiência.

Figura 1: Experiências de projeto baseadas em lei construtural.

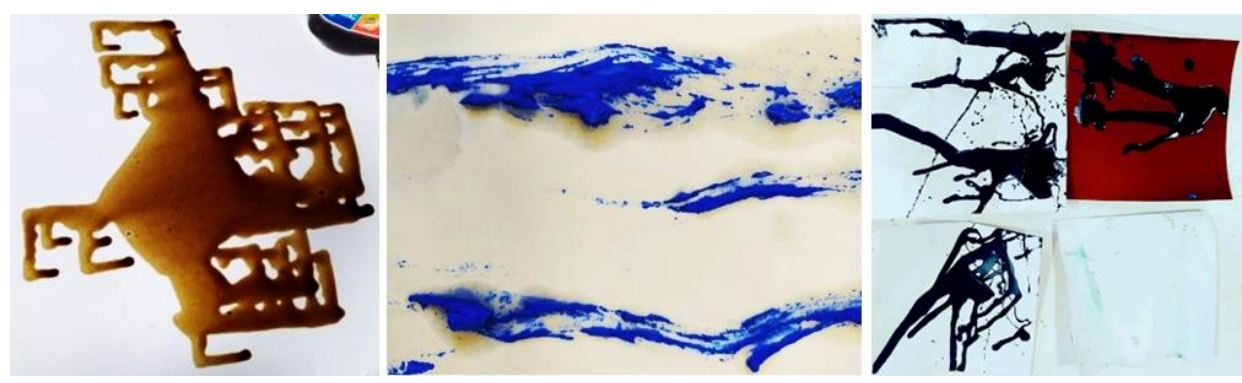

Fonte: Arquivo fotográfico da cadeira Morfología 2 B, FAUD-UNC (2019).

Figura 2: Experiências de projeto baseadas em sistemas de ramificação.
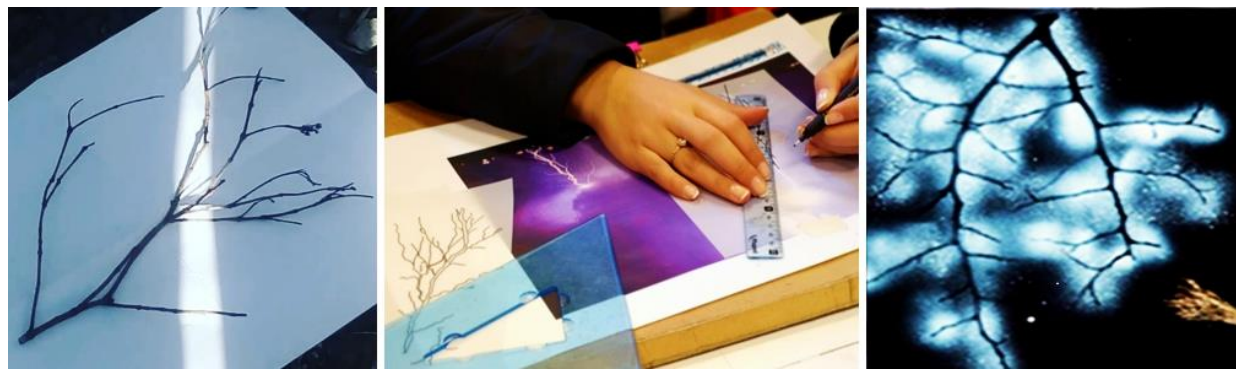

Fonte: Arquivo fotográfico da cadeira Morfología 2 B, FAUD-UNC (2019).

\section{Cinemática e cimática}

Em segundo lugar, vamos para o campo do conhecimento externo da física, a partir de seus ramos: cinemática e cimática - ambas coincidem no estudo de fenômenos periódicos - . A cinemática, enquadrada na física 
mecânica, concentra-se no estudo das trajetórias dos movimentos dos corpos no espaço, sem prestar atenção às forças que os produzem. Descreve e analisa diferentes tipos de movimentos corporais: retilíneo (uniforme e uniformemente acelerado), harmônico simples (movimento pendular), harmônico complexo (como uma combinação de movimentos harmônicos simples), movimentos circulares e parabólicos. A cimática, que se situa entre a acústica e a física, se concentra no estudo dos princípios de natureza acústica, mecânica e ótica que afetam a matéria. Segundo Lega Lladós (2015, p. 124), é a "...ciência que estuda as representações visuais das ondas sonoras e os fenômenos periódicos de vibração sobre a matéria".

Como aplicação destes princípios em experimentos projetuais e para fins didáticos, no caso da cinemática, são utilizados pêndulos simples carregados com substâncias líquidas ou sólidas de partículas finas (tintas e sal) que traçam trajetórias de movimentos em superfícies planas. Os movimentos oscilatórios representam trajetórias com diversidade e complexidade geométrica, que são delineadas em suportes de papel. Por outro lado, e com referência à cimática, são realizados ensaios com alto-falantes, nos quais superfícies planas (rígidas ou elásticas) são dispostas para conter sólidos de grão fino ou líquidos viscosos. O som, como um fenômeno periódico, transmite vibrações sob a forma de ondas mecânicas através da matéria. Embora estes eventos não ocorram de maneira idêntica, ocorre permanentemente em estado de vibração ou oscilação, o que torna possível registrar figuras (através de fotografias) que revelam padrões como resultado das variações de frequência.

Nestas pesquisas, são obtidos diversos conjuntos de trajetórias, que colocam em evidência o valor da experimentação com artefatos mecânicos e sonoros, que tornam viável a representação simultânea do movimento em estruturas gráficas.

Figura 3: Experiências de projeto baseadas em cinemática.
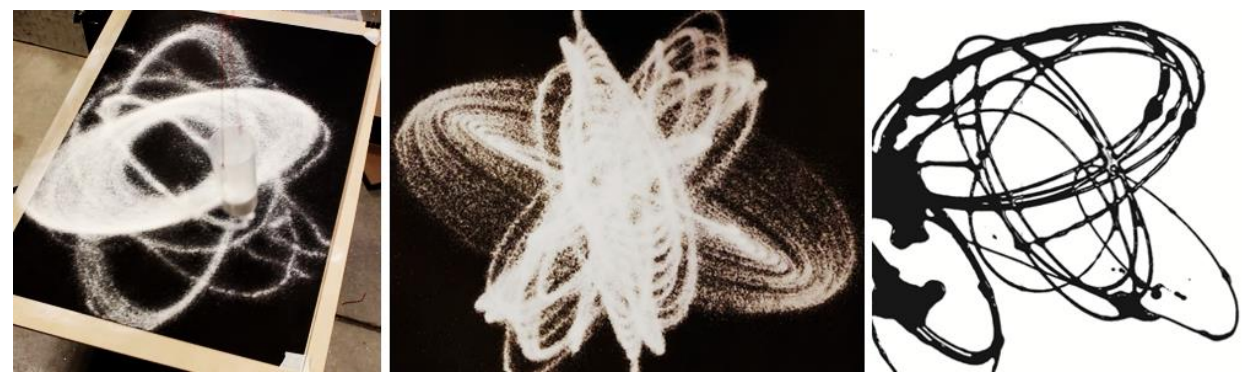

Fonte: Arquivo fotográfico da cadeira Morfología 2 B, FAUD-UNC (2019).

Figura 4: Experiências de projeto baseadas em cimática.
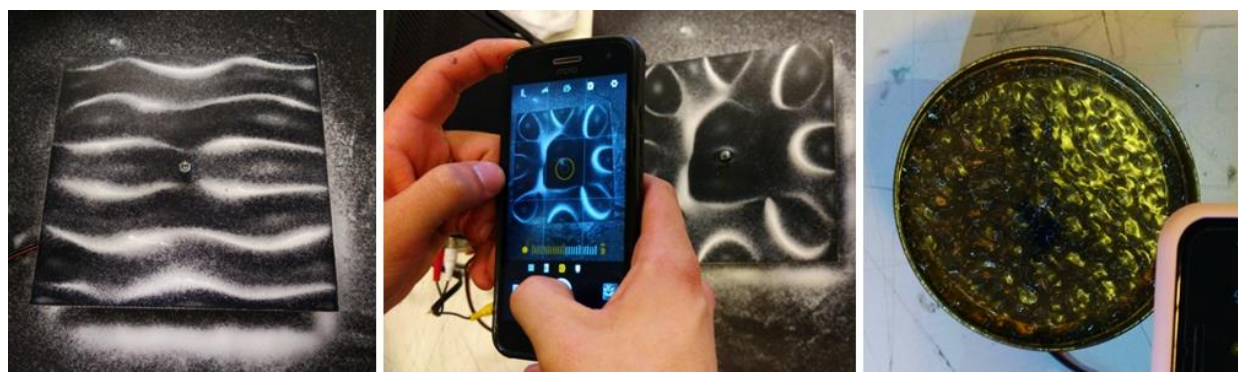

Fonte: Arquivo fotográfico da cadeira Morfología 2 B, FAUD-UNC (2019).

\section{Teoria dos jogos}

Como última linha de exploração é apresentada a teoria dos jogos, que corresponde a uma área da matemática aplicada, encarregada do estudo das estruturas formalizadas dos jogos e seus movimentos; é particularizada nos aspectos relativos à tomada de decisão como resposta à proposta de conflitos e se concentra em situações cujas características tornam possível sua análise e proposta de resolução através da modelagem da situação como um jogo abstrato. As estratégias e ações participam como variáveis determinantes das estruturas geométricas que representam o desenvolvimento dos jogos - as suas traduções em diagramas - . Os mesmos podem ser representados graficamente em uma matriz ou de forma normal e extensa ou sob a forma de uma árvore. Também é possível encontrar representações que apontem ações particulares ou movimentos individualizados. 
Em termos de experiência acadêmica, são propostos exercícios - com base nesta teoria — para geração de traçados reguladores, a partir do ensaio de jogos com regras estipuladas, que são planejados especificamente para experimentação, e que envolvem a ação física do grupo de estudantes em um campo espacial. Isto permite a modelização do registro das trajetórias de movimento geradas a partir de ações lúdicas e de acordo com as regras propostas e estipuladas. A particularidade da representação é que a mesma ocorre simultaneamente ao desenvolvimento do jogo, por meio de fitas que materializam as trajetórias daqueles que participam do jogo. Outra peculiaridade reside na construção síncrona e em escala real do espaço do jogo; desta forma, as estruturas geométricas tornam-se ferramentas de análise e questionamentos relacionados às pessoas - suas dimensões, proporções, escalas, tempos de movimento - desde sua origem.

Figura 5: Experiências de projeto baseadas na teoria dos jogos.
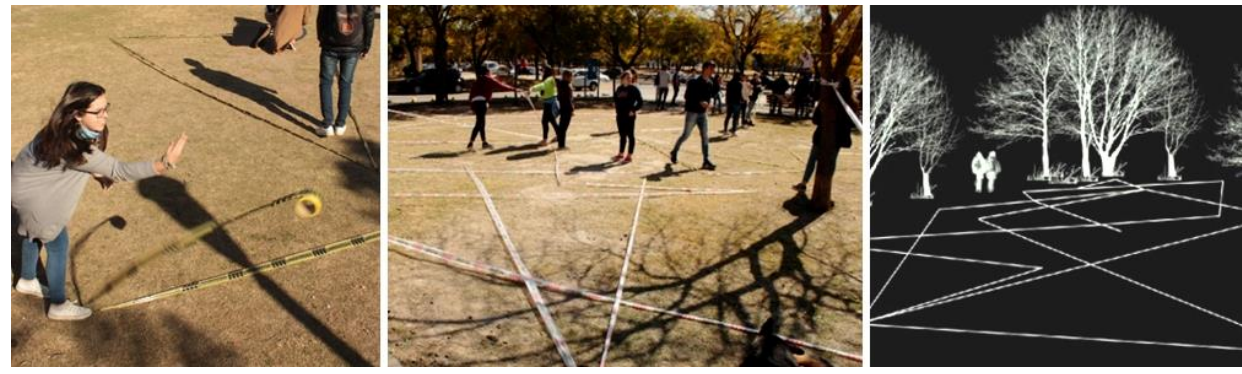

Fonte: Arquivo fotográfico da cadeira Morfología 2 B, FAUD-UNC (2019).

\section{Transferência de saberes como meio de produção}

Com base nas cinco linhas temáticas delineadas, são desenvolvidos ensaios que têm como objetivo pesquisar os múltiplos campos disciplinares. Os princípios generativos estudados e os padrões geométricos experimentados são considerados como possibilidades alternativas de pesquisa e geração morfológica, que proporcionam, particularmente, princípios organizacionais e generativos nas etapas iniciais dos processos de projeto.

Dentro da estrutura clássica do processo de design, dividido em três fases ou subprocessos: A) processo de ideação ou invenção do objeto de design, B) processo de projeção ou materialização profissional do objeto de design e C) processo de materialização ou construção do objeto de design - como uma obra arquitetônica - (NASELLI, 1999); a exploração é restrita na primeira das instâncias propostas. Este subprocesso de ideação, que, conforme definido por Naselli (2013, p. 55), "...implica a tradução das formas expressivas de ideação, imagens e conceitos, pré-arquitetônicos e estes para formas de aparência e significados arquitetônicos...". A fase mencionada é a fase seguinte ao desenvolvimento detalhado de um projeto, e permite a gestão e a prefiguração da forma para sua posterior evolução projetual. Esta gestação e respectiva configuração, neste caso, é guiada pela transferência de saberes caracterizada como um exercício trasdisciplinar.

Cada uma das diferentes experiências - em termos de técnicas e ferramentas utilizadas - que são desenvolvidas nas comissões de trabalho, obtém como produto preliminar uma série de estruturas geométricas ${ }^{2}$ que são materializadas em imagens capturadas por registro fotográfico, representação gráfica (design) ou como resultado do mesmo processo generativo de natureza gráfica (os ensaios que operam com tintas).

Estas estruturas são posteriormente replicadas e ajustadas em escalas de trabalho e evoluem como traçados reguladores ${ }^{3}$, incorporando uma lógica de ordenação. Diferentes tipos de traçados podem ser reconhecidos de acordo com a estrutura da qual se originam e as ações processuais que são aplicadas. Neste caso, operamos com seis tipos de traçados, correspondentes à seguinte classificação - resultado de uma pesquisa anterior da equipe (PERIES, 2018) -:

- Distorção: desequilíbrio ou torção das linhas que compõem uma trama para gerar traçados complexos, derivados dos estudos de Alberto Durero 4 .

- Scalling (no idioma inglês, referente à ação de ajustar as escalas): ampliação e/ou redução de estruturas sobrepostas com possíveis deslocamentos para gerar traçados complexos, derivados dos estudos de Peter Eisenman.

- Distribuição: união de pontos por vínculos para gerar traçados de rede complexos, derivados dos estudos de Paul Barán sobre redes de comunicação.

- Triangulação: a união de três pontos por enlaces ou a união de planos triangulares por adjacência para gerar traçados complexos de rede ou tessela, derivados do procedimento de Boris Delaunay.

- Regionalização: circunscrição dos planos que geram um traçado de tessela, derivado do procedimento de Gueorgui Voronói. 
- Fluxo: sucessão de pontos que fluem no espaço e definem trajetórias para gerar traçados complexos, derivados dos conceitos de Zaha Hadid.

Com relação às explorações da lei construtural e sistemas de ramificação, é necessário estabelecer traçados associados ao fluxo e à distribuição, que correspondem aos trajetos de fluidos e aos princípios de crescimento vegetal, neuronal e descargas elétricas, ordenando as trajetórias e nós de união para estabelecer redes.

Figura 6: Síntese de traçados resultantes da exploração de lei construtural e sistemas de ramificação.
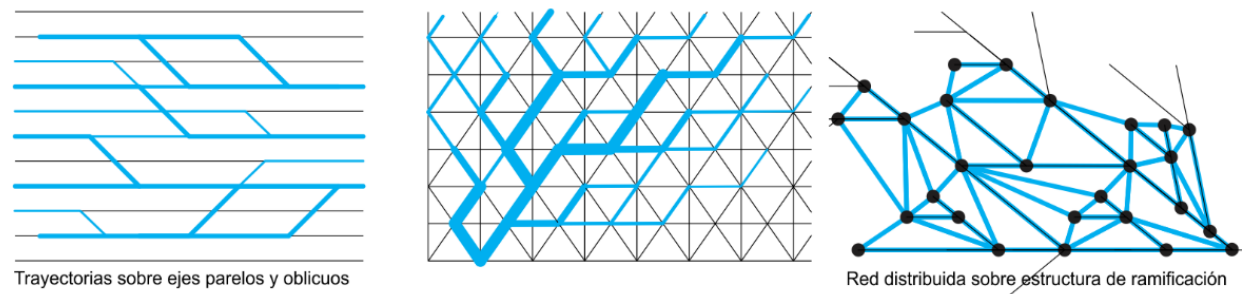

Fonte: Arquivo fotográfico da cadeira Morfología 2 B, FAUD-UNC (2019).

As experiências que transferem os princípios da cinemática obtêm estruturas gráficas com ordem geométrica intrínseca, coerentes com a lógica mecânica dos dispositivos pendulares que as geram. Neste caso, as estratégias para a construção de traçados apelam para a superposição de esquemas de trajetórias, para a ampliação ou redução de fragmentos e para os deslocamentos, a fim de construir traçados reguladores sob a lógica de scalling. Quanto às práticas que emergem das linhas temáticas de cimática, as ondas sonoras e os fenômenos periódicos de vibração da matéria são predominantemente expressos em traçados do tipo distorção que expressam as ondas sonoras e as vibrações sobre a matéria.

Figura 7: Síntese de traçados resultantes da exploração de cinemática e cimática.
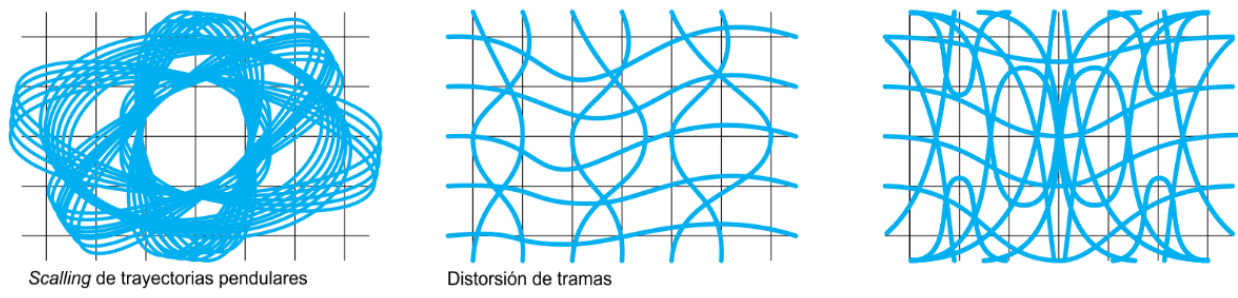

Fonte: Arquivo fotográfico da cadeira Morfología 2 B, FAUD-UNC (2019).

Nas explorações baseadas na teoria dos jogos, cada sucessão de passos ou movimentos é registrada com pontos que fluem no espaço e definem trajetórias. Três modalidades são adotadas para a construção dos traçados: A- a regionalização e triangulação, desde a tradução das trajetórias lineares até tesselas triangulares ou poligonais, que representam as regiões de movimento ou o setor de ação daqueles que jogam. B- a distribuição, a partir da união das linhas convergentes em esquemas de rede, que é construída a partir da representação de quem joga como um nó e seus possíveis passes ou circulações como linhas ou artistas C- o fluxo, a partir da disposição das próprias linhas da trajetória. Em todas as alternativas, a repetição dos esquemas é contemplada para contribuir com a complexidade dos traçados.

Figura 8: Síntese de traçados resultantes da exploração de teoria de jogos.
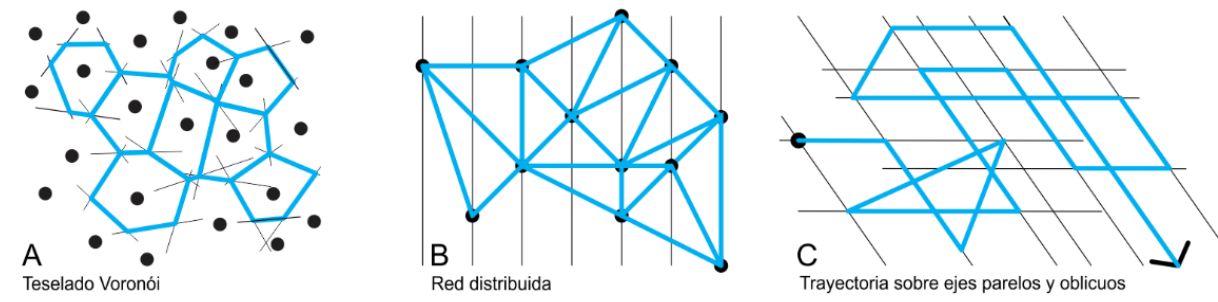

Fonte: Arquivo fotográfico da cadeira Morfología 2 B, FAUD-UNC (2019).

O precedente descreve os casos em que opera com representações bidimensionais. O procedimento a seguir apresenta o desafio de abordar as questões tridimensionais. Cada estudante, com seu próprio traçado regulador, tem a possibilidade de replicar os princípios do padrão gerador ou encontrar uma lei ou mecanismo, com lógica homóloga, que lhe permita produzir o crescimento da estrutura geométrica nas três dimensões espaciais. O trabalho simultâneo com modelos físicos e representações gráficas (digitais e analógicas) produz 
formas que são alojadas no espaço tridimensional. Nas etapas subsequentes, surgem abordagens da espacialidade arquitetônica, que estão em sintonia com os princípios generativos e trazem as condições de seus axiomas e métodos. Os traçados reguladores são mantidos presentes para orientar e dar precisão às ações que superponham, justaponham, encastrem, subtraiam ou ampliem figuras e elementos, aqueles que progressivamente prefiguram os arquétipos arquitetônicos primários - parede, teto, piso, porta, janela - .

O desenvolvimento do projeto leva ao design dos limites espaciais a partir do design do material dos invólucros. Com esta ação, de condição evolutiva, a espacialidade adquire forma como protoarquitetura, para chegar à fase de fechamento do processo de ideação de um pavilhão de exposições. Os procedimentos envolvem diversificações ligadas às intenções e interesses de cada estudante, como mostram as ilustrações a seguir, que resumem, de forma representativa, o processo de três equipes de estudantes.

Como resultado do exercício de projeto, existem coincidências associadas às condições determinadas por "transferências disciplinares", e desacordos quanto à direção dos processos de projetos pessoais. Nesse sentido, a amplitude e diversidade das propostas que emergem dos objetivos e diretrizes comuns são particularmente valorizadas. Em uma avaliação geral - dos 220 trabalhos realizados em grupos de 2 estudantes do ciclo de 2019 - é reconhecida a diversidade e heterogeneidade das alternativas, aquelas que respondem ao desafio experimental proposto e são uma consequência direta da metodologia implementada.

Figura 9: Trabalhos representativos das explorações do projeto.
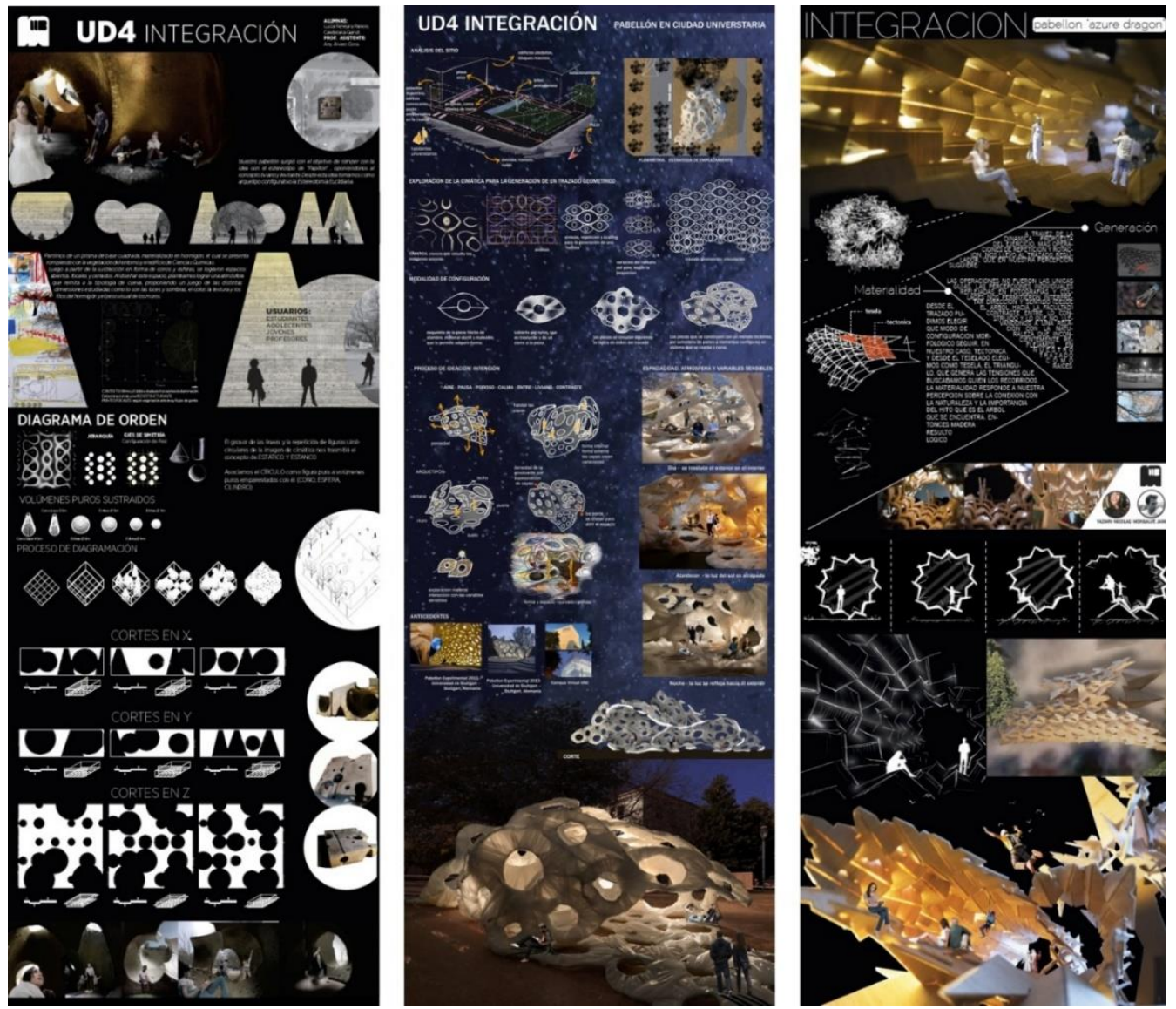

Fonte: Os estudantes (1) Ferreyra Panero L. e Garlot C, (2) Gjurkan M. V. e Godoy-Magallán B. e (3) Nicolas J. e Monsalve J. (2019).

\section{CONCLUSÃO}

Em referência à proposta de especificar práticas que favorecem a exploração e sistematização das ordens geométricas, mencionamos os contextos de conhecimento que viabilizam a estruturação e organização formal. Pode-se identificar que as abordagens de experimentação de projetos a partir da mistura de saberes contribuem para a realização de produções que também relacionam e integram conhecimentos. Ao mesmo tempo, favorecem a continuidade das linhas de pesquisa que estão sendo enfrentadas e demarcam aberturas para o conhecimento contingente. 
Com relação às disciplinas escolhidas, é evidente que a metodologia abordada beneficia a identificação de padrões com potencial para ensaios e sistematização, através de práticas disciplinares de pesquisa. A contribuição da "disciplinaridade cruzada" é valorizada como uma abordagem baseada na tração para o campo do conhecimento da morfologia; neste caso, as particularidades das experiências direcionam e influenciam a geração da forma arquitetônica.

Com a dinâmica trasdisciplinar exposta, é construída uma bagagem de informações múltiplas, com um caráter conceitual e operacional, que "nutre" o processo de projeto e faz contribuições únicas. Este conjunto de conhecimentos torna-se uma desculpa ou um pretexto para desenvolver práticas experimentais criativas e espontâneas, enquanto são arbitrados e regulados pelos códigos e axiomas correspondentes a cada campo disciplinar em que é questionado.

Todo o fenômeno global que cada participante desenvolve - como personalidade criativa - é constituído na causa e no fundamento que desencadeia a produção morfológica e, simultaneamente, contribui na aquisição de valores e na formação de atitudes e aptidões como profissionais do projeto. Outra das contribuições corresponde à aproximação dos estudantes ao exercício de pesquisa, no qual eles desenvolvem suas primeiras práticas formais, uma ação que favorece a possibilidade de despertar vocações. Considera-se que cada proposta é nutrida por esta abordagem trasdisciplinar e trascultural, e como Herţeg, Pioariu e Popescu (2011, p. 7) declaram "...pesquisam problemas, oferecem e provam soluções a partir de uma perspectiva curricular transversal"

No que diz respeito aos aspectos metodológicos, a possibilidade de vincular o ensino à pesquisa é particularmente valorizada, e de passar por um exercício integrado, no qual os resultados preliminares da pesquisa básica são combinados, enquanto são transferidos para o campo educacional para ensaios práticos e formação de estudantes. Da mesma forma, as produções da experiência acadêmica são transferidas para o projeto científico para sua avaliação e validação como uma construção do conhecimento original.

\section{REFERÊNCIAS}

ALARCÓN, C.; CARBONELL, V.; HOTT, D.; MAGENDZO, A.; MARFÁN, J. ¿Cómo trabajar los Objetivos Fundamentales Transversales en el Aula?. Santiago: Governo do Chile, Ministério de Educação, 2003.

ARNAUD, P. J. L.; MANIEZ, F.; RENNER, V. Cross-Disciplinary Perspectives on Lexical Blending. Berlín: De Gruyter Mouton, 2012.

BANCHIO, I. La transdisciplina en el proceso de ideación arquitectónico, desde un enfoque morfológico (Tese de mestrado). Universidade Católica de Córdoba, 2012.

BERTOGLIA, E. M. Complejidad, transdisciplinariedad y competencias: Siete viñetas pedagógicas. Valencia: Letreme editorial, 2019.

DE PABLOS PONS, J. La visión disciplinar en el espacio de las tecnologías de la información y comunicación. En Tecnologías para transformar la educación. Tecnologías para transformar la educación de Juana María Sancho Gil (coord.) (Escritor) pp. 77-102, 2006.

DI CASTRI, F.; HADLEY, M. Enhancing the credibility of ecology: Is interdisciplinary research for land use planning useful?. Geo Journal 13, pp. 299-325, 1986.

DOBERTI, R. Espacialidades. Buenos Aires: Ediciones Infinito, 2008.

HERTEG, C., PIOARIU, R.; POPESCU, T. Cross-disciplinary Approaches to the English Language: Theory and Practice. Cambridge Scholars Publishing, 2011.

LLADÓS, F. L. Creación sonora sobre fluidos. Barcelona, Research, Art, Creation, 3(2), pp. 122-158, 2015. DOI: 10.17583/ brac.2015.1390

LITWIN, E. El oficio de enseñar. Condiciones y contextos. Buenos Aires: Paidós, 2016.

MARTINEAU, J. (Ed.). Quadrivium: Las cuatro artes liberales clásicas. 2014 - Sciencia: Matemáticas, Física, Química, Biología y Astronomía. 2014. - Designa: Los secretos técnicos de las artes visuales tradicionales. 2016 - Geomancia: Dragones, feng shui, líneas ley, radiestesia y misterios de la tierra. 2016 - Trivium: Las artes liberales clásicas de gramática, lógica, retórica. 2016 - Megalitos: Estudios en piedra. 2019. Madri: Librero.

NICOLESCU, B. La transdisciplinariedad. Manifiesto. México: Edición 7 Saberes, 1996.

MUÑOZ, M. T. La mirada del otro. Madri: Ediciones Asimétricas, 2010.

NASELLI, C. A. El proceso de diseño como concepto instrumental. En N. Goytia, Cuando la idea se construye, pp. 28-32. Córdoba: Screen, 1999. 
NASELLI, C. A. El rol de la innovación creadora: en la lógica interna del diseño arquitectónico. Córdoba: I+P y EDUCC, 2013.

PAVA-GÓMEZ, A. J.; BETANCUR-VILLEGAS, M. A.; PÁEZ-CALVO, A. Planteamiento de una estrategia desde la construcción de una investigación proyectual. Revista de Arquitectura, 20 (1), 88-101, $2018 . \quad$ DOI: http://dx.doi.org/10.14718/revarq.2018.20.1.1954

PERIES, L. La experimentación proyectual en la enseñanza de la morfología arquitectónica. En I. Moisset y L. Períes (Comp.), La experimentación proyectual: actas VIII Projetar 2017, pp. 534-547. Buenos Aires: UBA, 2017.

PERIES, L. Órdenes geométricos complejos en la construcción de trazados reguladores. En A. Pollet (Comp.), VII Jornadas de Investigación "Encuentro y Reflexión", pp. 35-42. Córdoba: FAUD-UNC, 2018.

POKROPEK, J. La experimentación proyectual en la enseñanza: Enseñar a construir sentido. Cuadernos del Centro de Estudios en Diseño y Comunicación, 82, pp. 115-126, 2020.

QUINTANILLA, M. A.; VIDAL, J. Specialisation and Cross-disciplinarity Patterns and the Design of New Higher Education Programmes. Higher Education Management, 12, 2, pp. 53-63, 2000. DOI: https://dx.doi.org/10.1787/hemp-v12-2-en

SANCHO GIL, J. (coord). Tecnologías para transformar la educación. Sociedad, cultura y educación. Madri: Akal, 2006.

SANTOMÉ, J. T. Globalización e interdisciplinariedad: el curriculum integrado. Madri: Morata, 1994.

SARQUIS, J.; BUGANZA, J. La teoría del conocimiento transdisciplinar a partir de Manifiesto de Basarab Nicolescu en Fundamentos en Humanidades, vol. X, núm. 19. pp. 43-55, 2009.

\section{NOTAS}

${ }^{1}$ Tradução do autor. "A given researcher will consider himself to be a specialist in a particular scientific field but in order to work in that area he needs to master and to be able to use methods and results coming from several different disciplines."

2 Entende-se por estrutura a distribuição e a ordem lógica dos componentes geométricos (pontos, linhas, planos).

${ }^{3}$ Os traçados reguladores são uma estrutura geométrica com uma certa lógica organizacional e processual.

${ }^{4} \mathrm{Na}$ definição de cada tipo de traçado, é incorporado um autor/a de referência, dado que a operação geométrica com a qual se intervém ou a lógica organizacional deriva do trabalho dessas personalidades.

5 Tradução da autora. “...investigate problems, offer and probe solutions from a cross-curricular perspective.”

NOTA DO EDITOR $\left(^{\star}\right)$ : O conteúdo do artigo e as imagens nele publicadas são de responsabilidade do(s) autor(es). 